American Journal of Environmental Sciences 2 (3): 92-94, 2006

ISSN 1553-345X

(C) 2006 Science Publications

\title{
Contributions To The Study Of Heavy Metals Concentration Variation In Sedimentable Dusts According To The Distance From The Pollution Source
}

\author{
Mariana DOBRA, Vasile VIMAN and Gheorghe VÂTCĂ \\ North University of Baia Mare, V. Babeş st. no. 62/A \\ 430083 Baia Mare, Romania
}

\begin{abstract}
The environment in areas where geological and mining activities, such as the extraction of ores containing heavy metals, take place, is heavily polluted with dusts resulting from these activities, as well as with residual waters from the mines. Depending on the meteorological conditions, as well as on the conditions under which sedimentable dusts or dusts in suspension are emitted into the air, the distance from the main pollution source varies considerably. In order to estimate the pollution level and the danger presented by this phenomenon, some analyses are required with regard to determining the concentration of heavy pollutant metals in air, soil and plants samples, as well as in dusts from the air. For the precise determination of the major components, as well as the minor ones, and also the ones in traces, the analytical techniques used must have low detection limits and the lowest matrix effects possible ${ }^{[1,2]}$. The methods that respond to these requirements are from the category of inductively coupled plasma atomic emission spectrometry (ICP-AES). The research was done in an area where there are industrial units whose main field of activity is extraction of certain ores which contain $\mathrm{Pb}, \mathrm{Cu}$ and $\mathrm{Zn}$ as major components, as well as $\mathrm{Cr}, \mathrm{Mn}, \mathrm{Ni}, \mathrm{Co}, \mathrm{Ag}, \mathrm{Au}, \mathrm{Al}$, and $\mathrm{Fe}$ as minor components or in traces. It is obvious that the presence of these metals in the air, water and soil has a negative impact on human health, plants and animals. This paper is a study of these aspects in an area where pollution with heavy metals reaches alarming quotas.
\end{abstract}

Keywords: heavy metals, ICP-AES, speciation

\section{INTRODUCTION}

Different natural and artificial sources of pollution can pollute the environment with heavy metals. If natural sources contribute to this only to little extent, artificial ones, which result from different human activities, contribute decisively to the pollution of the environment. To be more specific, heavy metal artificial polluting sources are basically the following: the stationary incinerators for urban residues, the metallurgical industry, the materials for the construction industry, the mines and the plants for the enrichment of nonferrous ores into useful components.

According to the environment in which pollutants are emitted and to the type of combination in which they are found, the distribution and the mobility of heavy metals varies considerably. Thus, with the contribution of local meteorological factors and of the chemical form in which the metal is found, the heavy metals ( $\mathrm{Pb}, \mathrm{Cu}, \mathrm{Zn}, \mathrm{Al}, \mathrm{Fe})$ emitted into the atmosphere as dusts in suspension will be unequally dispersed around the pollution source.

\section{MATERIALS AND METHODS}

The sample collecting system depends on the nature of the environment from which they are collected. Mainly, the placement of the sample collecting points must regard the data obtained after the analysis of the samples which must offer an exact image on the concentrations of the pollutants in the area, meaning that the error in estimating the concentrations in places from where samples were not collected should be minimal and the results of the analysis should offer the possibility to estimate the contribution of the individual sources to the global field of the concentrations. The samples were collected by sedimentation in cylindrical vessels with $25 \mathrm{~cm}$ in diameter, placed on $1.5 \mathrm{~m}$ high supports, far from highways or building sites, in order to avoid contamination of samples.

To prepare the samples for analysis reagents with spectral purity were used. The sedimentation dust samples were dried and disintegrated in a CEM MDS 2000 microwave with a mixture of hydrochloric acid,

Corresponding Author: Mariana DOBRA, North University of Baia Mare, V., Romania 
fluorhydric acid and nitric acid in teflon containers. The disintegration lasted 10 minutes at $50 \%$ power and 120 psi pressure and it was followed by 10 minutes of oxidation. The solutions were transfered in balloons of $50 \mathrm{ml}$ and brought to sign with bidistilled water.

The samples of dusts in suspension together with the filter were rendered soluble with nitric acid, sulphuric acid and perchloric acid, the work method being specific for every metal to be determined.

The obtained solutions were submitted to analysis in order to determine the concentrations of heavy metals, the analysis being made with a BAIRD ICP 2070 spectrometer, which was calibrated with a standard Merck solution. The spectrometer operated at a power of $900 \mathrm{~W}$, a flux of the cooling gas of $91 \mathrm{Ar}$ $\mathrm{min}^{-1}$ and an auxiliary flux of 1,5 $1 \mathrm{Ar}^{\mathrm{min}} \mathrm{mi}^{-1}[3,4,5]$.

\section{RESULTS AND DISCUSSION}

Before starting the experimental determination it is necessary to carry out the wavelength calibration at which we make the metal determinations, with the BAIRD ICP 2070 spectrometer. For the concentration determination of the following metals $\mathrm{Pb}, \mathrm{Cu}, \mathrm{Zn}, \mathrm{Fe}$, $\mathrm{Al}$, from sedimentable powders, the measurement was done at the following wavelengths: $\mathrm{Pb}-220,35$ (nm); Cu-324.75 (nm); Zn-213.86 (nm); Fe-238.20 (nm); Al$396.15(\mathrm{~nm})$.

After that, we analyzed each sample to determine the content in heavy metals. The concentrations of metals in the analyzed samples are showen in fig. 1 . The data from fig. 1 show maximum concentrations for $\mathrm{Fe}, \mathrm{Cu}, \mathrm{Zn}$ in sample 2 at $1.5 \mathrm{Km} \mathrm{W}$ far from the main polluting source. $\mathrm{Pb}$ reaches the maximum concentration in sample 1 at $0.5 \mathrm{Km} \mathrm{W}$, and $\mathrm{Al}$ reaches the maximum in sample 9 at $30 \mathrm{~km} \mathrm{~W}$.

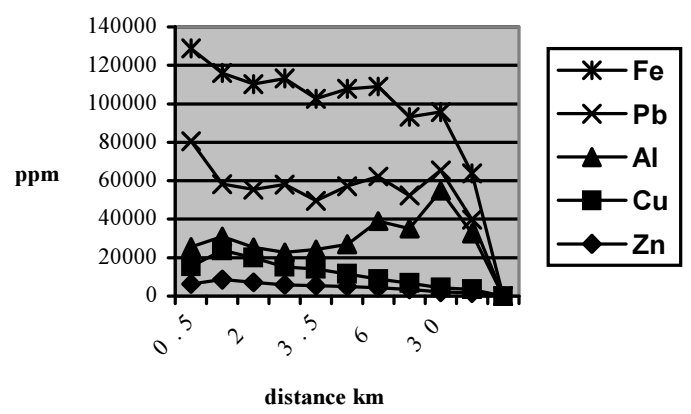

Fig.1: Variation of heavy metals concentration according to distance

The experimental findings can be explained only if we consider the emission rates of the pollution sources, geographic, meteorological and geological factors, which contribute to the dispersion and accumulation of pollutants, the nature and characteristics of the soil and of the chemical combinations in which metals are found.

The pollutant emissions are transported to different distances, depending on the geographic area, its geology and the local meteorological factors: fog, wind, rains, thermal inversions.

All these natural factors of environment, specific to the area, do not contribute to a fast dispersion of pollutants and thus, the level of momentary pollutant concentrations is not reduced, because in situations of atmospheric instability, pollutants are transported to highly polluted areas, and in situations of atmospheric calm, the diffusion of pollutants is very weak.

Geographically, the researched area is limited at north and east by hills with heights of $600-700 \mathrm{~m}$; at east, at a greater distance, their height increases to $1400 \mathrm{~m}$. Plain zones predominate at south and west. This will determine a circulation of the air mainly on southeast, northwest and west directions; on these directions, high concentrations of metals are also found. A small speed of the wind, of $2,2 \mathrm{~m} / \mathrm{s}$ in $70 \%$ of cases and an atmospheric calm with a frequency of $49 \%$ will determine a weak ventilation of the area and the dusts from the air will deposit on short distances from the main pollution sources, thus determining a strong pollution of the soil.

The medium annual rains of $800-1400 \mathrm{~mm}$ involve the sedimentable dusts and the dusts in suspension from the air and they deposit them on the soil.

The thermal inversion produced at a height of approximately $500 \mathrm{~m}$, acts like a barrage stopping the diffusion of pollutants on height, which would lead to the decrease of momentary concentrations on soil. The thermal stratification of the air is unstable; this fact determines the wedge of pollutants to be wave shaped, the wastes being quickly mixed into the surrounding air, and thus higher concentration values for pollutant elements in soil are obtained close to the pollution sources.

The local meteorological factors that were analyzed do not create favorable conditions for quick dispersion of pollutants, so that high concentrations appear on a small area around the pollution source, mainly in points situated in the most frequent directions of the wind.

The chemical combination types in which the metals are found, their solubility and molecular mass can explain the distribution of the pollutants, experimentally found. To identify the chemical combination types, speciation analyses of $\mathrm{Pb}, \mathrm{Cu}$ and 
$\mathrm{Zn}$ were done. In sedimentable powders, the following percentages of $\mathrm{Pb}$ were found: sulphides $47 \%$, oxides and carbonates $18 \%$ and sulphates $35 \%$

The high molecular mass and the low solubility of the combinations limit the mobility of $\mathrm{Pb}$ and they achieve high concentrations closer to the polluting source.

In sedimentable powders, $\mathrm{Cu}$ was identified as $17 \%$ sulphate, $34 \%$ oxid and carbonate, $49 \%$ sulphide. Considering the percentage over 69 of the $\mathrm{Cu}$ unsoluble combinations in sedimentable dusts, we can explain their low mobility and the distribution of $\mathrm{Cu}$ in high quantities at distances of up to $6 \mathrm{~km}$ for the pollution source.

For $\mathrm{Zn}$ there were identified in sedimentable powders $39 \%$ sulphide, $31 \%$ sulphate, $16 \%$ silicate and $14 \%$ oxide and carbonate. The increased amount of sulphate in dusts, compared to the ores, is explained by the partial transforming of sulphide oxide under the influence of sulphuric acid drops which exist in the atmosphere in the area, strongly polluted with sulphur dioxide. This compositional structure correlated with local meteorological factors explains it. $\mathrm{Zn}$ distribution is in the greatest amount when closer to the main polluting source.

Using a graphics programme for the experimentaly obtained data, we find, for example (figure 2), that the variation of $\mathrm{Pb}$ concentration according to distance takes place according to the following law: $\ln y=a+b x / \ln x$.

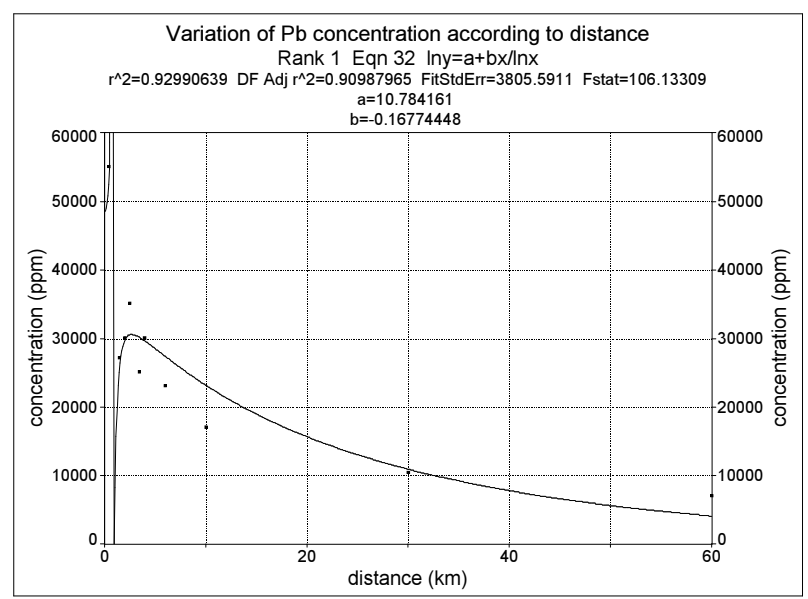

Fig. 2 Variation of $\mathrm{Pb}$ concentration according to distance

\section{CONCLUSION}

The frequency of different diseases caused by $\mathrm{Pb}$ is higher in this area with up to $90 \%$ as reported to the unpolluted areas, the agricultural crops are smaller with $50-60 \%$ and the forest losses reach $50 \%$.

Generally, the heavy metals concentration is 3-8 times higher in plants from the researched area, as compared to plants collected from unpolluted areas.

By determining the concentration of heavy metals in sedimentable dusts, with the help of ICP-AES method, we can appreciate that the Baia Mare area is strongly aggressed by anthropic activity, thus taking one of the record places in cumulative pollution in Europe.

The use of the ICP-AES method of analysis for the determination of heavy metals concentration of other pollutants as well, allow efficient measures to be taken for ecological reconstruction.

\section{REFERENCES}

1. Thompson, M., 1985. The capabilities of inductively coupled plasma atomic emission spectrometry, Analyst, 110, 443-449

2. Thompson, M. and Walsh J. N. (1983). A Handbook of Inductively Coupled Plasma Spectrometry, Blackie, Glasgow, 83-116, 197 228.

3. Barnes, R.M., Schleicker, R.G., 1975. Spectrochimica Acta, vol 30 B, PP 109-134

4. Baumans, P.W.J.M., 1987. Inductively Coupled Plasma Emission Spectroscopy, M.X. Wiley Ed., PP 69-99

5. Montaser, A., Golightly, D.W.1987. Inductively Coupled Plasmas in Analytical Atomic Spectrometry, VCH Publishers Ed., PP 163-198; 267-319; 604-621 